SHORT REPORT

\title{
Secular trends in waist circumference in Spanish adolescents, 1995 to 2000-02
}

\author{
L A Moreno, A Sarría, J Fleta, A Marcos, M Bueno
}

Arch Dis Child 2005;90:818-819. doi: 10.1136/adc.2004.067066

Two studies, in 1995 and 2000-02, were compared to assess changes in waist circumference in adolescents. Between the two time periods, waist circumference increased significantly in males at 13 years and in females at 14 years. Significant changes in waist circumference were observed during the study period; the rates of change were 0.53 and $0.86 \mathrm{~cm} / \mathrm{y}$ in boys and 0.67 and $0.87 \mathrm{~cm} / \mathrm{y}$ in girls. Future morbidity in adolescents may be affected due to accumulation of excess central fat.

A bdominal adiposity is a component of the metabolic syndrome, already observed in children and adolescents. ${ }^{1}$ Whereas the best methods for estimating abdominal fat are imaging techniques, an anthropometric surrogate (waist circumference) is used for population studies. For children and adolescents, data on temporal changes in abdominal adiposity are scarce. In British youth, McCarthy and colleagues ${ }^{2}$ have observed trends in waist circumference that greatly exceeded those in BMI, particularly in girls. To establish preventive measure to fight childhood obesity and its consequences there is a need to monitor total and abdominal adiposity in children and adolescents. The aim of this study was to assess recent changes in waist circumference in Spanish adolescents.

\section{METHODS AND RESULTS}

In this study we consider data from two cross-sectional studies, both representative of Zaragoza (Spain). The first was conducted in 1995; ${ }^{3}$ for this analysis we have included 233 adolescents (aged 13-14 years; 128 boys and 105 girls). The second was conducted within the framework of the AVENA Study, from January 2000 to March 2002; for this analysis, we have included 162 adolescents (aged 13-14 years; 72 boys and 90 girls). The protocol of the AVENA Study was approved by the Review Committee for Research Involving Human Subjects of the Hospital Universitario Marqués de Valdecilla (Santander, Spain).

Weight and height were measured and the body mass index (BMI) was calculated. Waist circumference was measured with an inelastic tape: the subject was in a standing position, and the tape was applied horizontally midway between the lowest rib margin and the iliac crest, at the end of gentle expiration; ${ }^{4}$ technical error of measurement was $0.95 \mathrm{~cm}$, and reliability $98.0 \%{ }^{4}$ The technical error of measurement was obtained by carrying out a number of repeated measurements on the same subject, by the same observer; the coefficient of reliability reveals what proportion of the between-subject variance in a measured population is free from measurement error.

For each survey we calculated means and standard deviations by sex and age. Comparisons were done by the Mann-Whitney U test. Multiple regression analysis was used to assess whether the effect of time in waist circumference changes was independent of the change in total adiposity assessed by the BMI; independent variables included in the regression model were BMI and the time when the waist measurements were taken.

Table 1 shows changes in BMI and waist circumference in Zaragoza from 1995 to 2000-02. For waist circumference, absolute changes were significant in all the groups, except at 13 years in males; parallel changes were observed for BMI. For waist circumference, the rate of increase was $0.53 \mathrm{~cm} / \mathrm{y}$ at 13 years and $0.86 \mathrm{~cm} / \mathrm{y}$ at 14 years in boys, and $0.67 \mathrm{~cm} / \mathrm{y}$ at 13 years and $0.87 \mathrm{~cm} / \mathrm{y}$ at 14 years in girls. Regression models showed that changes in waist circumference during the studied period greatly depend on total adiposity assessed by the BMI (significant effect in all the age and sex groups, with $\mathrm{p}<0.0001$ in all cases). However, time period had a significant effect in males at 13 years $(p=0.022)$, and in females at 14 years $(p=0.001)$. The full regression models are available on request.

\section{DISCUSSION}

We found a trend to higher waist circumference values (central adiposity) in adolescents from Zaragoza aged 13-14 years, from 1995 to 2000-02. These changes were related to changes in total adiposity (BMI), but changes in waist circumference (abdominal adiposity) were independent of BMI changes, at least in some age groups. The observed changes in waist circumference measurements in the two cross-sectional studies were quite large; however, results in each survey and sex and age group also showed an important between-subject variability. The results observed in Zaragoza can be extrapolated to the rest of Spain, because Zaragoza is a representative city of the rest of the country.

Data on waist circumference changes are available only in British adolescents. ${ }^{2}$ In 11-16 year old British adolescents, waist circumference increase rate was $0.345 \mathrm{~cm} / \mathrm{y}$ in boys and $0.62 \mathrm{~cm} / \mathrm{y}$ in girls. ${ }^{2}$ The authors hypothesised that levels of physical activity have decreased faster in males than in females, and central fatness may be related more to physical activity than to energy intake. The rate of increase in our adolescents was similar to that observed in British girls.

It has also recently been observed in British children aged 2-5 years that mean waist circumference values were greater in 1995-98 that in a previous study conducted in $1987 .{ }^{5}$ The proportional increase in waist circumference for each age-sex group generally exceeded the proportional increase in BMI.

The increase in waist circumference in our study probably reflects both visceral and subcutaneous fat and hence total fatness. The accumulation of excess fat predominantly in the upper body rather than in the peripheral region has been previously observed in our population. ${ }^{3}$ From the scarce data in children and adolescents, it seems that paediatric populations of European countries experience a 


\begin{tabular}{|c|c|c|c|c|c|}
\hline & \multicolumn{2}{|l|}{1995} & \multicolumn{2}{|c|}{$2000-02$} & \multirow[b]{2}{*}{$\mathbf{p}$} \\
\hline & Mean & (SD) & Mean & (SD) & \\
\hline \multicolumn{6}{|l|}{ BMI $\left(\mathrm{kg} / \mathrm{m}^{2}\right)$} \\
\hline \multicolumn{6}{|l|}{ Males } \\
\hline 13 years & 19.66 & (2.94) & 20.25 & (3.43) & 0.436 \\
\hline 14 years & 20.53 & (2.93) & 22.10 & (4.14) & 0.076 \\
\hline \multicolumn{6}{|l|}{ Females } \\
\hline 13 years & 20.04 & (3.11) & 21.76 & (3.64) & 0.004 \\
\hline 14 years & 20.03 & (2.40) & 21.40 & (2.83) & 0.027 \\
\hline \multicolumn{6}{|c|}{ Waist circumference $(\mathrm{cm})$} \\
\hline \multicolumn{6}{|c|}{ Males } \\
\hline 13 years & 70.62 & (7.65) & 73.83 & (10.24) & 0.226 \\
\hline 14 years & 72.75 & (6.78) & 77.90 & (11.89) & 0.049 \\
\hline \multicolumn{6}{|l|}{ Females } \\
\hline 13 years & 67.29 & (6.44) & 71.28 & (7.90) & 0.005 \\
\hline 14 years & 66.81 & (5.02) & 72.04 & (6.88) & 0.001 \\
\hline
\end{tabular}

trend to increased general and abdominal adiposity. Current and future morbidity in our adolescents may be seriously affected due to accumulation of excess central fat. Prevention programmes should be tailored for use beginning in childhood.

\section{Authors' affiliations}

L A Moreno, J Fleta, E.U. Ciencias de la Salud, Universidad de Zaragoza, Zaragoza, Spain

A Sarría, M Bueno, Departamento de Pediatría, Universidad de Zaragoza, Zaragoza, Spain

A Marcos, Grupo Inmunonutrición, Departamento de Nutrición y Metabolismo, Consejo Superior de Investigaciones Científicas, Madrid, Spain

Funding: The AVENA study was supported by the Spanish Ministry of Health (FIS 00/0015 and Red de Centros C03/09), and grants from Panrico S.A., Madaus S.A., and Procter and Gamble S.A.

Competing interests: none
Correspondence to: Prof. L A Moreno, E.U. Ciencias de la Salud, Universidad de Zaragoza, Domingo Miral s/n, 50009 Zaragoza, Spain; Imoreno@unizar.es

Accepted 22 February 2005

\section{REFERENCES}

1 Moreno LA, Pineda I, Rodríguez G, et al. Waist circumference for the screening of the metabolic syndrome in children. Acta Paediatr 2002;91:1307-12.

2 McCarthy HD, Ellis SM, Cole TJ. Central overweight and obesity in British youth aged 11-16 years: cross sectional surveys of waist circumference. BMJ 2003;326:624-6.

3 Moreno LA, Fleta J, Sarría A, et al. Secular changes in body fat patterning in children and adolescents of Zaragoza (Spain), 1980-1995. Int J Obes 2001;25:1656-60.

4 Moreno LA, Joyanes M, Mesana MI, for the AVENA Study Group, et al. Harmonization of anthropometric measurements for a multicenter nutrition survey in Spanish adolescents. Nutrition 2003;19:481-6.

5 McCarthy HD, Jarrete KV, Emmett PM, Rogers I, and the ALSPAC Study Team. Trends in waist circumference in young British children: a comparative study. Int J Obes Relat Metab Disord 2005;29:157-62. 